\title{
Análisis y aplicación de los suelos arcillosos residuales de características expansivas
}

\author{
L. Zeevaert-Wiechers \\ División de Estudios de Posgrado, Facultad de Ingeniería, UNAM
}

(recibido: marzo 1999; aceptado: diciembre de 2000)

\begin{abstract}
Resumen
En múltiples lugares de la corteza terrestre existen suelos residuales con propiedades expansivas, los que en general se encuentran con grados muy bajos de saturación. En los suelos residuales de características expansivas, el aumento en el grado de saturación provoca reducción de la adherencia entre los granos e hinchamiento del mineral de arcilla, ocasionándose cambios importantes en el comportamiento mecánico. Lo anterior provoca deformaciones irregulares en la superficie de los suelos y en las construcciones que se apoyan sobre ellos. De ahí la necesidad de conocer los parámetros cuantitativos que rigen el comportamiento estático y dinámico de este tipo de suelos en relación con los cambios ambientales de humedad.
\end{abstract}

Descriptores: suelos residuales; expansivos; comportamiento mecánico; parámetros cuantitativos

\section{Abstract}

In many places of the earth crust there are fine residual soils with expansive features. In general, these soils are found with very low degrees of saturation. Residual soils of expansive characteristics, the increase in the degree of saturation produces a reduction in the bond between the grains and the swelling of the clay mineral takes place, inducing important changes in the mechanical behavior such as irregular vertical displacements of the ground surface and of the engineering constructions on top of it. It is therefore, necessary to learn the quantitative parameters that govern the static and dynamic behavior of this type of soils with respect to the moisture-related environmental changes.

Keywords: residual soils; expansive; mechanical behavior; quantitative parameters.

\section{Prólogo}

En múltiples lugares de la corteza terrestre existen suelos residuales expansivos que, en general, se encuentran con grados bajos de saturación y un alto porcentaje de minerales activos de arcillas.

Cuando aumenta el grado de saturación, el mineral de arcilla sufre un hinchamiento, el cual se registra en la superficie del suelo por distorsiones o alabeamiento. Las obras que se apoyan sobre estos suelos sufren daños importantes; de ahí, la importancia de conocer los parámetros cuantitativos del comportamiento mecánico estático y aún dinámico de este tipo de suelos expansivos que se presentan con diferentes características mecánicas debido al ambiente donde se formaron.

La investigación de estos suelos es importante y necesaria en materia de mecánica de suelos y deberá efectuarse en los diferentes tipos de suelos residuales que se encuentran en la naturaleza. Las características de expansión dependen principalmente de varios factores físico-químicos que son propiedad de las arcillas que los constituyen. Sin embargo, existen modalidades similares del comportamiento mecánico para todos estos suelos.

Este trabajo pretende interesar a los ingenieros estudiosos, demostrando la experiencia del autor y exponiendo el resultado de sus investigaciones para crear motivación y así permanezca el desarrollo de la investigación y tecnología básica racional de los suelos expansivos residuales con varias características ambientales.

\section{Recapitulación de los suelos residuales}

La corteza terrestre se encuentra ocupada en un alto porcentaje por suelos residuales que generalmente no se 
manifiestan saturados; algunos están constituidos por un alto porcentaje de minerales de arcilla, limo, arena fina y un bajo grado de saturación. Los climas áridos generalmente contienen carbonatos de calcio. (Zeevaert, 1982).

Se hace hincapié en que todos los suelos responden con expansión al ser descargados de los esfuerzos iniciales y volumétricos a los que están confinados, esto es, con el esfuerzo octaédrico con que se encuentran en la naturaleza. Cuando éstos no contienen minerales activos se espera una respuesta de expansión elástica, como es el caso de los suelos que contienen minerales de caolinita y los suelos saturados (Zeevaert, 1982 y Terzaghi, 1941).

En los suelos saturados lacustres o marinos que contienen minerales activos del grupo de las montmorilonitas que son ávidas a la adhesión molecular del agua, se espera con el tiempo una expansión por hinchamiento, como resultado del aumento del espesor de los minerales y el incremento en las películas de agua adherida entre granos, que a su vez, es ocasionado por la expansión elástica, con la correspondiente reducción del término de resistencia a la cohesión.

Por un cambio en las condiciones ambientales y construcciones puede aumentar el grado de saturación debido a la falta de evaporación o corrientes de agua en el subsuelo que provocan el hinchamiento en los minerales de arcilla, ocasionando resistencias bajas y compresibilidades altas, que corresponden a la alteración que sufre el suelo por el aumento en el grado de saturación.

Al ser hidratadas las arcillas expansivas, la compresibilidad aumenta y la resistencia al esfuerzo cortante disminuye, por tanto, este fenómeno es de importancia práctica para el ingeniero de cimentaciones (Zeevaert, 1982 y 1991).

Las arcillas que presentan estos fenómenos con mayor intensidad son las que contienen minerales del grupo de las montmorilonitas, principalmente las de litio, sodio o calcio, en donde las pertenecientes al litio son más activas. Consecuentemente, las obras que se construyen sobre estos suelos pueden sufrir deterioro importante (Grim, 1953).

Por consiguiente, el ingeniero de suelos está obligado a investigar los parámetros mecánicos que permitan valorar según el caso que se presente, los desplazamientos verticales que tal vez sean probables por el aumento en el grado de saturación.

\section{Observación en campo}

La primera vez que el autor tuvo que tratar el estudio con suelos arcillosos residuales de tipo expansivo fue en el año de 1951, en ciudad Obregón, Sonora, para la cimentación de dos baterías de silos, así como instalaciones de poco peso.

Los análisis de expansión calculados con el resultado de las pruebas de laboratorio de la arcilla fueron verificados en el campo por el arquitecto Gustavo F. Aguilar por medio de placas de $1 \times 1 \mathrm{~m}$ apoyadas en lentes de arcilla expansiva, conocida en la región como "Barrial", la cual se encuentra intercalada con sedimentos eólicos finos.

Por observaciones de campo se comprende la preocupación de los constructores en las obras civiles que se construyen en la región, debido a los múltiples daños que se observan en calles, banquetas, construcciones de poco peso y rotura de las instalaciones de ductos.

El barrial de la región estudiada, es una arcilla con poco limo y arena fina que contiene carbonatos de calcio y está constituida por minerales de montmorilonita de calcio. El grado de saturación es muy bajo, ya que el espejo de agua superficial se encuentra a $23 \mathrm{~m}$ de profundidad, al hidratarse la arcilla por falta de evaporación o por impregnación de agua en el subsuelo se hincha en forma considerable (Rey, 1982).

Otras arcillas que presentan características expansivas pueden contener montmorilonita de sodio, o bien, litio que es la más activa, éstas corresponden a las arcillas lacųstres típicas de la cuenca del valle de México que por estar saturadas presentan expansión elástica al ser relevadas o disminuidas del esfuerzo octaédrico de campo y solamente a través del tiempo se adiciona la expansión por el fenómeno de hinchamiento.

El tipo de mineral se puede detectar por medio de análisis químicos y térmicos; sin embargo, lo más importante es conocer sus propiedades mecánicas de expansión y los parámetros mecánicos correspondientes que permitan realizar cálculos del comportamiento del subsuelo.

Se han realizado otras investigaciones en suelos expansivos; por ejemplo, en las arcillas residuales de Coro, Colombia (Sánchez, 1979) en donde se muestra una alta potencia de expansión. En este material se efectuaron pruebas a esfuerzo vertical y volumen constantes; las últimas reflejan el efecto de relajamiento del esfuerzo generado por la hidratación.

La interpretación de los resultados de las investigaciones realizadas por el autor y la supervisión en los análisis de las arcillas expansivas, se reportan a continuación, así como la explicación de la metodología para llevar a cabo las pruebas en el laboratorio.

\section{Análisis mecánico de laboratorio}

Para estimar la magnitud mecánica de las propiedades de expansión de estos suelos en el estado de confinamiento que se encuentran en la naturaleza, se puede utilizar la prueba del odómetro con las modalidades que requiere la investigación.

El odómetro es el símil adecuado para representar las condiciones de confinamiento de campo. Para realizar esta prueba se requiere una probeta de suelo inalterado que no 
haya sufrido alteración en su microestructura o cambio en su grado de saturación natural, la pastilla que constituye la probeta y el anillo para confinarla debe tener una relación diámetro a altura no menor de ocho. Las piedras porosas que se utilicen deben contener únicamente la humedad que les proporciona el ambiente.

Para detectar el fenómeno natural de la potencia de expansión, se coloca la probeta de suelo inalterado en el odómetro y se le aplican incrementos iguales hasta un esfuerzo determinado; para el cual, se requiere conocer la potencia de expansión. En esta condición se permite que la probeta se hidrate por capilaridad, colocándole agua gradualmente y haciendo que suba el nivel de ésta lentamente, de abajo hacia arriba, hasta llegar a la piedra porosa superior, permitiendo así la hidratación del suelo. A su vez, se lleva a cabo un registro de la expansión contra el tiempo. Cuando ya no se registre hinchamiento se procede a continuar la prueba aplicando nuevamente incrementos de carga iguales, hasta obtener una curva que registre con claridad la compresibilidad del suelo ocasionado por el fenómeno de expansión.

La figura 1 muestra la configuración de la compresibilidad que toma el suelo durante la prueba para esfuerzo vertical constante.
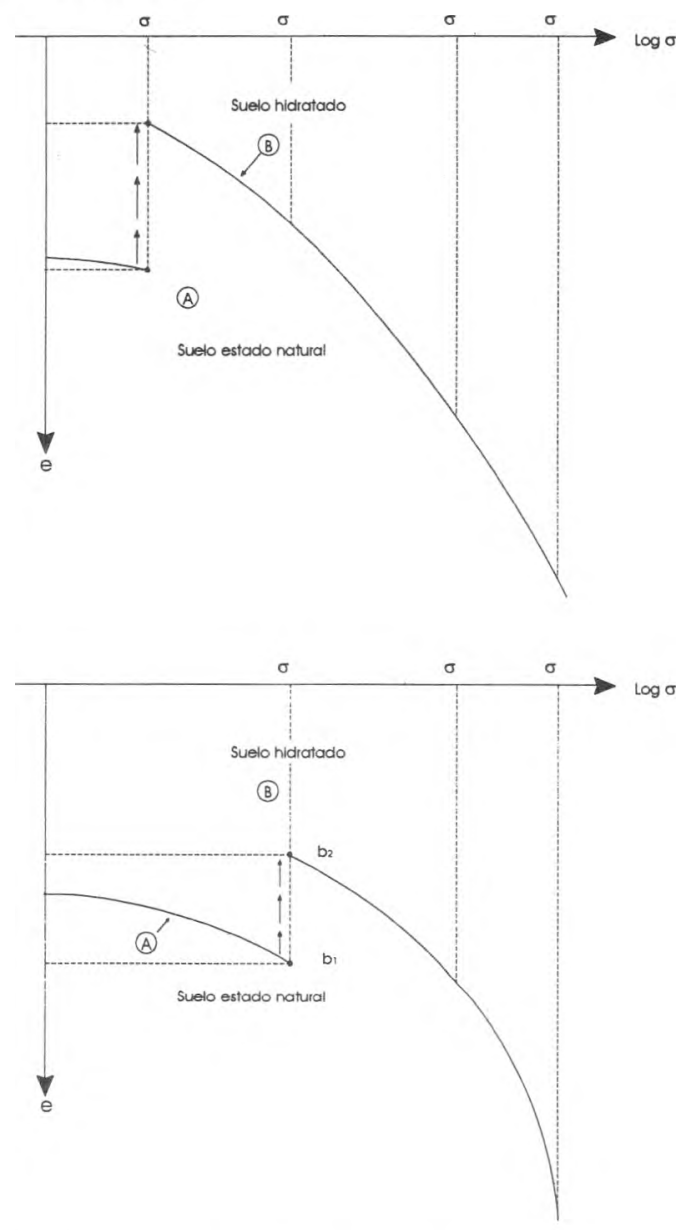

Figura 1. Expansión para un determinado confinamiento

\section{Conclusiones de los análisis de laboratorio}

De múltiples pruebas efectuadas en el laboratorio del suscrito y en la DEPFI, UNAM, realizadas en suelos con características de expansión, se llega a la conclusión de que la magnitud del hinchamiento o expansión del suelo está comprendido entre las siguientes dos curvas de compresibilidad (Fig. 2), la curva A representa la compresibilidad del suelo inalterado en su estado natural y la curva B representa la compresibilidad del suelo expansivo previamente hidratado por capilaridad en el odómetro con un esfuerzo vertical inicial de $0.10 \mathrm{~kg} / \mathrm{cm}^{2}$, antes de iniciar la prueba.

De lo anterior, se concluye que cuando se trata de una hidratación del suelo con alto contenido de minerales de arcilla con fuerte actividad, se demuestra que al hidratarse el suelo para varios valores del confinamiento dado por $\left(\sigma_{z}\right)$ la expansión alcanza la curva $\mathbf{B}$ del suelo pre-hidratado, y al resumir el proceso de carga se obtiene la compresibilidad que muestra la curva B (Fig. 2).

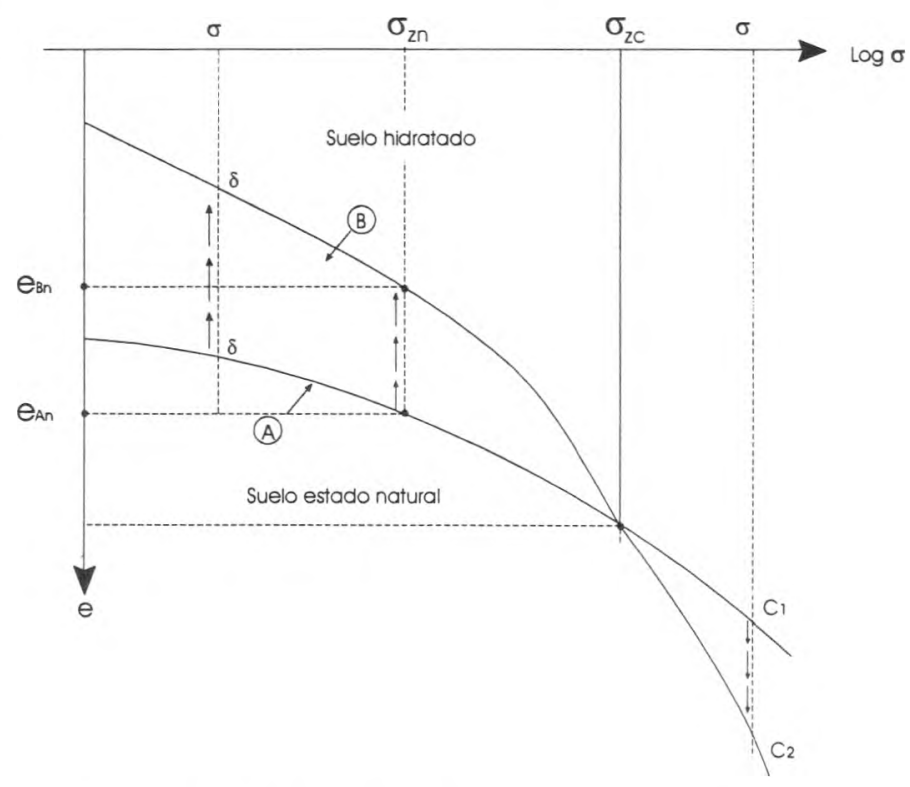

Figura 2. Compresibilidades para suelo en estado natural y previamente hidratado

En el caso de que el contenido de arcilla en el suelo sea una fracción más pequeña con respecto al contenido de limo y arena fina que contiene el suelo, entonces la expansión resulta de un valor intermedio entre las compresibilidades dadas por las curvas A y B, esto es por la curva C (Fig. 3). Así también, para hidratación incompleta se obtienen los valores que se sitúan entre las dos curvas antes mencionadas.

De las curvas (figura 2 y 3 ) se puede establecer la deformación unitaria máxima de la expansión para un valor determinado del esfuerzo $\left(\sigma_{z \mathrm{n}}\right)$ como sigue:

$$
\varepsilon_{\mathrm{sn}}=-\left(\mathrm{e}_{\mathrm{An}}-\mathrm{e}_{\mathrm{Bn}} /\left(1+\mathrm{e}_{\mathrm{An}}\right)\right.
$$




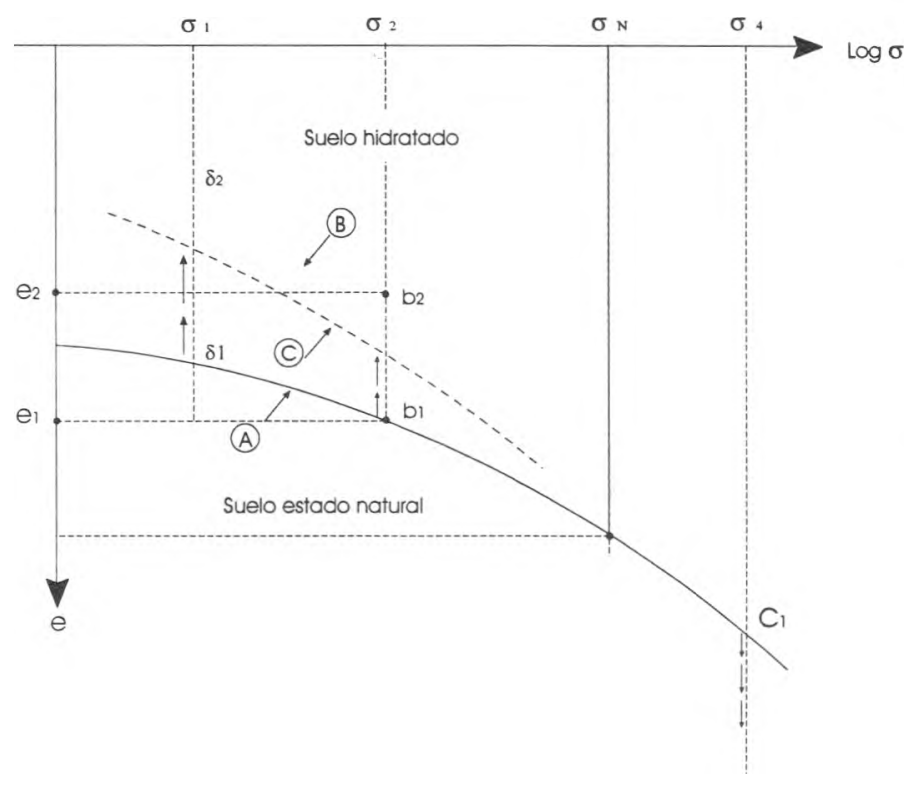

Figura 3. Suelo que presenta expansión incompleta curva $C$

El valor lineal de la expansión de la prueba para un esfuerzo vertical $\left(\sigma_{z \mathrm{n}}\right)$ se calcula multiplicando la ecuación 1 por el espesor (d) de la probeta

$$
\Delta \delta \mathrm{n}=-\Delta \varepsilon_{\mathrm{sn}}(\mathrm{d})
$$

De lo anterior, se obtiene también el coeficiente de expansión volumétrica unitaria por medio de la ecuación

$$
\mathrm{m}_{\mathrm{ze}}=-\varepsilon_{\mathrm{sn}} / \sigma_{\mathrm{zn}}
$$

Nótese de la figura 2 que las curvas $\mathbf{A}$ y $\mathbf{B}$ se cortan en un punto $\sigma_{z c}$, lo que significa que para este esfuerzo no existe expansión ni compresión y que a partir de este valor sólo se verifica la compresión que muestra la curva $\mathbf{B}$ para suelo pre-hidratado que tiene mayor compresibilidad que la que muestra la curva A para el suelo en estado natural.

De lo anterior, se concluye que el ingeniero de mecánica de suelos puede contar con un procedimiento racional para estimar la expansión o hinchamiento de los suelos residuales de características expansivas que por alguna razón sufran un cambio ambiental o generado por el hombre.

\section{Aplicación de resultados de laboratorio al campo}

Para utilizar los resultados de los análisis de laboratorio, con el objeto de estimar los desplazamientos verticales en el campo, se hace necesario conocer la siguiente información (Zeevaert, 1980):
1. La estratigrafía del lugar en cuestión, hasta la profundidad que indique el diagnóstico preliminar del problema de que se trate.

2. Los esfuerzos efectivos de sobrecarga con la profundidad, adicionados de los que produzca la construcción.

3. Conocer el tipo de cimentación, su geometría y la carga unitaria que arroja ésta sobre el suelo al nivel de desplante.

4. La oquedad y el grado de saturación con la profundidad.

5. Conocer el módulo lineal de deformación unitaria de los estratos con arcillas expansivas en el perfil estratigráfico.

La relación que existe entre el módulo de expansión lineal de deformación unitaria y el coeficiente de expansión volumétrica unitario se puede calcular por medio de la siguiente ecuación (Zeevaert, 1982):

$$
\begin{gathered}
\mathrm{m}_{z \mathrm{e}}=-\mathrm{vcM}_{z \mathrm{e}} \quad \text { también } \\
\mathrm{M}_{z \mathrm{e}}=-\mathrm{m}_{\mathrm{ze}} / \mathrm{vc}
\end{gathered}
$$

En donde Mze es el módulo lineal de deformación unitaria vertical de la expansión y (v) la relación de Poisson. En las arcillas expansivas se puede usar $v=0.40$. El valor de (vc) se estima por medio de la siguiente fórmula (Zeevaert, 1982):

$$
\mathrm{vc}=(1+\mathrm{v})(1-2 \mathrm{v}) /(1-\mathrm{v}), \text { aprox. }=0.62
$$

Conociendo la información citada anteriormente se puede calcular el levantamiento vertical que se ocasiona en la superficie del suelo o en las cimentaciones por el fenómeno de la expansión o hinchamiento que propician las arcillas residuales por el aumento del grado de saturación (Zeevaert, 1980).

A continuación se presenta cómo llevar a cabo el cálculo en forma tabular de los levantamientos verticales ocasionados por la hidratación de estos suelos que se encuentran en la naturaleza con grados bajos de saturación y así poder decidir sobre la elección del método para reducir la expansión por el hinchamiento de los minerales de arcilla (Tabla 1).

La expansión por estrato es: $\Delta \delta_{\mathrm{e}}=\mathrm{Me}(\mathrm{d})(\sigma)$, en donde $\alpha_{\mathrm{e}}=\mathrm{M}_{\mathrm{e}}(\mathrm{d})$, es la expansión volumétrica de un estrato. Para un número $(\mathbf{n})$ de estratos

$$
\delta \mathrm{e}=\Sigma_{1}\{\alpha \mathrm{e}(\sigma)\} \mathrm{n}
$$

Los valores de $\mathrm{M}_{\mathrm{e}} \mathrm{y}(\sigma)$ se consideran valores medios de un estrato. 
Tabla 1. Disposición del cálculo de expansión

\begin{tabular}{cccccccccc}
\hline \hline & $\delta_{1}$ & $\delta_{2}$ & $\delta_{3}$ & $\delta_{4}$ & $\delta_{4}$ & $\delta_{3}$ & $\delta_{2}$ & $\delta_{1}$ & $\alpha$ \\
\hline \hline Estrato & $\mathrm{q} 1$ & & $\mathrm{q} 1$ & & & $\mathrm{q} 1$ & & $\mathrm{q} 1$ & \\
\hline $\mathrm{A}$ & $\sigma_{1} \mathrm{~A}$ & $\sigma_{2} \mathrm{~A}$ & $\sigma_{3} \mathrm{~A}$ & $\sigma_{4} \mathrm{~A}$ & $\sigma_{4} \mathrm{~A}$ & $\sigma_{3} \mathrm{~A}$ & $\sigma_{2} \mathrm{~A}$ & $\sigma_{1} \mathrm{~A}$ & $\alpha \mathrm{A}$ \\
& & & & & & & & & \\
$\mathrm{B}$ & $\sigma_{1} \mathrm{~B}$ & $\sigma_{2} \mathrm{~B}$ & $\sigma_{3} \mathrm{~B}$ & $\sigma_{4} \mathrm{~B}$ & $\sigma_{4} \mathrm{~B}$ & $\sigma_{3} \mathrm{~B}$ & $\sigma_{2} \mathrm{~B}$ & $\sigma_{1} \mathrm{~B}$ & $\alpha \mathrm{B}$ \\
& & & & & & & & & \\
$\mathrm{C}$ & $\sigma_{1} \mathrm{C}$ & $\sigma_{2} \mathrm{C}$ & $\sigma_{3} \mathrm{C}$ & $\sigma_{4} \mathrm{C}$ & $\sigma_{4} \mathrm{C}$ & $\sigma_{3} \mathrm{C}$ & $\sigma_{2} \mathrm{C}$ & $\sigma_{1} \mathrm{C}$ & $\alpha \mathrm{C}$ \\
& & & & & & & & & \\
$\mathrm{D}$ & $\sigma_{1} \mathrm{D}$ & $\sigma_{2} \mathrm{D}$ & $\sigma_{3} \mathrm{D}$ & $\sigma_{4} \mathrm{D}$ & $\sigma_{4} \mathrm{D}$ & $\sigma_{3} \mathrm{D}$ & $\sigma_{2} \mathrm{D}$ & $\sigma_{1} \mathrm{D}$ & $\alpha \mathrm{D}$ \\
\hline \hline
\end{tabular}

$$
\delta_{\text {en }}=(\alpha \sigma) n^{A}+(\alpha \sigma) n^{B}+(\alpha \sigma) n^{C}+\ldots \ldots .
$$

qn, Carga unitaria de cimentación sobre el suelo $\sigma \mathbf{n}^{\mathrm{N}}$, Representa el estado de esfuerzos verticales unitarios en la masa del subsuelo por peso propio y por la carga que origina la cimentación.

Dependiendo del orden de magnitud de los levantamientos verticales se pueden aplicar en forma racional las siguientes soluciones:

1. Zanjas perimetrales a la construcción que eviten el aumento de la humedad bajo la cimentación y construir pavimentos impermeables para evitar la penetración del agua en el subsuelo.

2. Ejercer el humedecimiento previo del subsuelo.

3. Zapatas aisladas cuando los levantamientos diferenciales sean tolerables (Fig. 4).

4. Zapatas continuas con trabes de rigidez (Figs. 5 y 6 ).

5. Losa corrida rigidizada (Fig 7).

6. Cimentación de tipo cajón rígido (Fig. 8).

7. Pilotes de fricción apoyados en la zona de suelo estable (Fig. 9).

8. Pilotes apoyados de punta en un estrato resistente (Fig. 10).

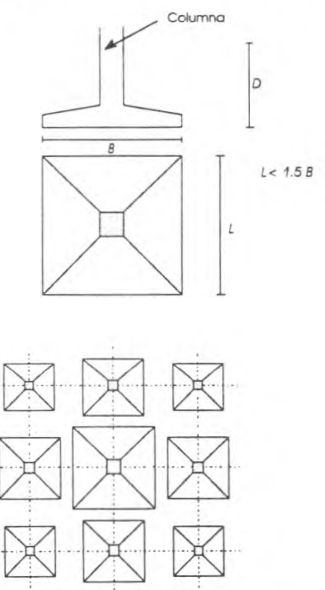

Figura 4. Zapatas aisladas
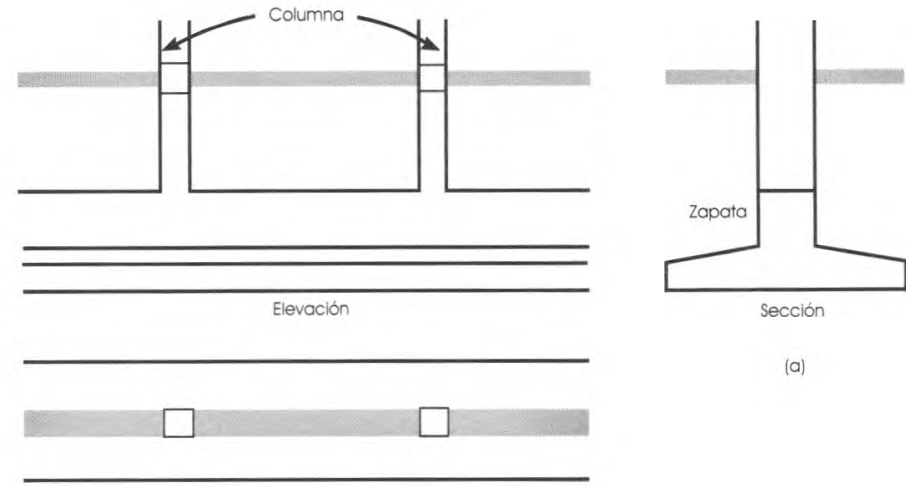

(a)

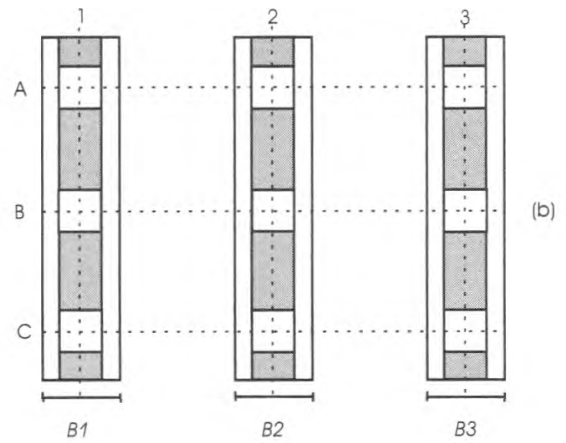

Figura 5. Zapatas continuas rigidizadas
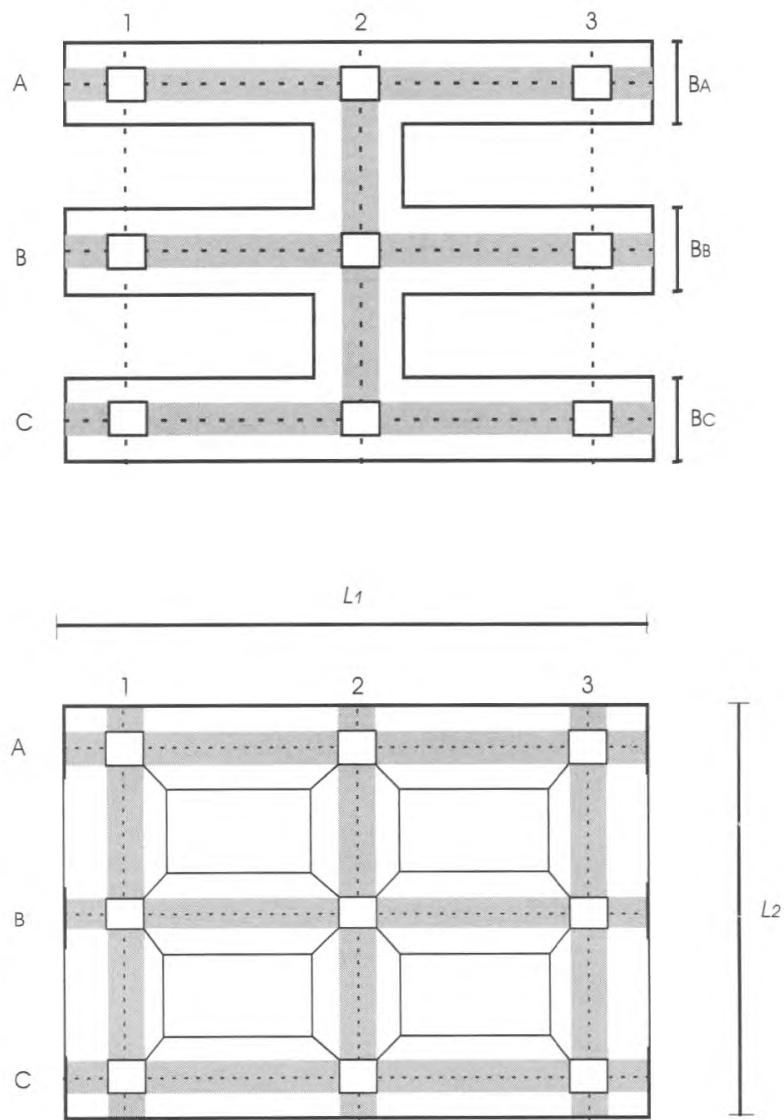

(b) 
DOI: http://dx.doi.org/10.22201/fi.25940732e.2001.02n1.005
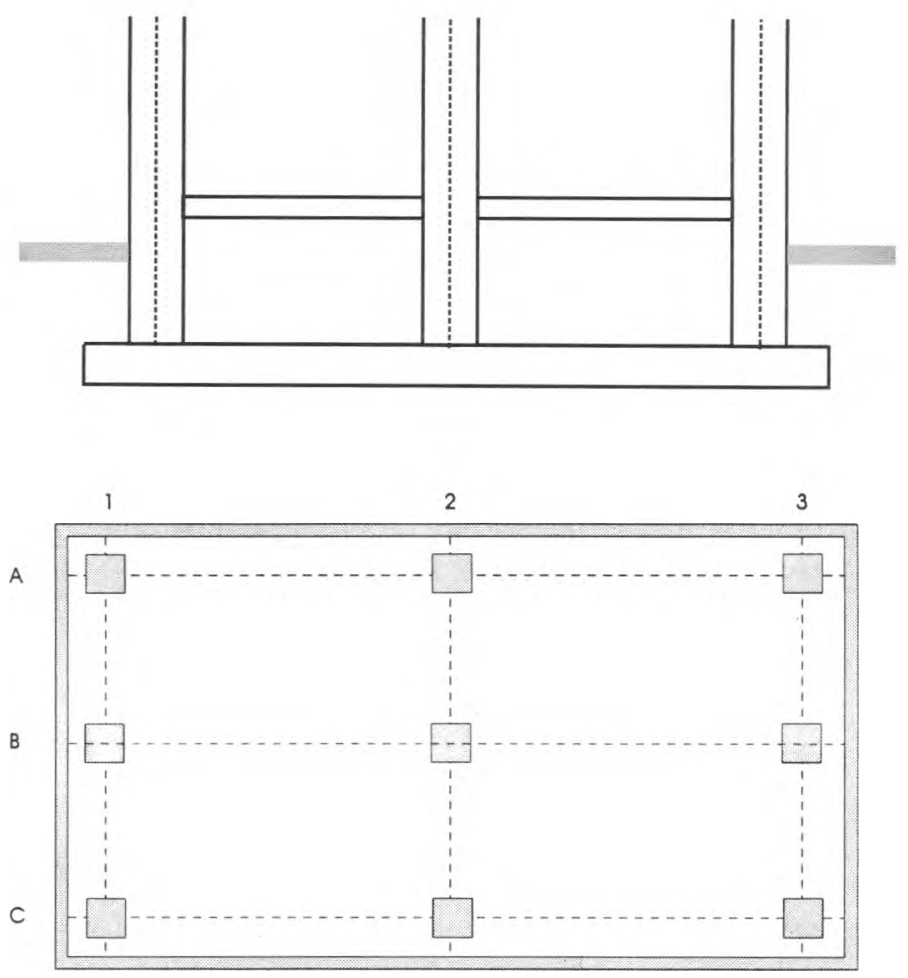

Figura 7. Losa rigidizada
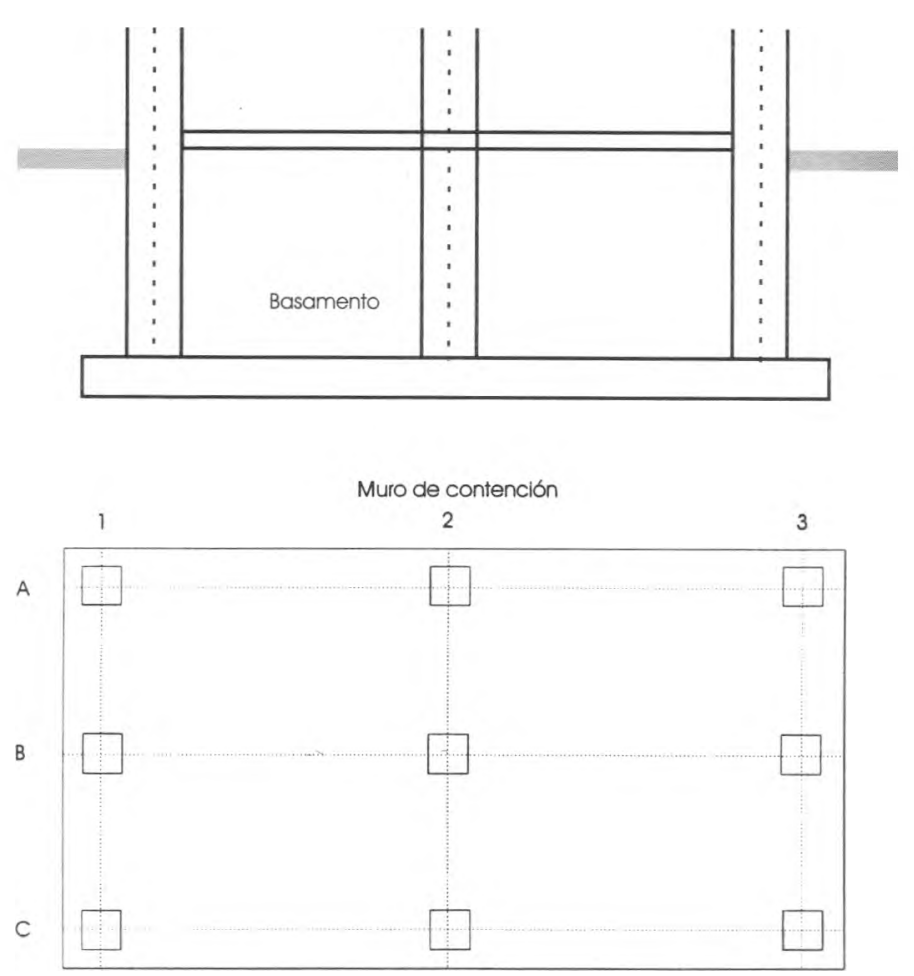

Figura 8. Cimentación de cajón
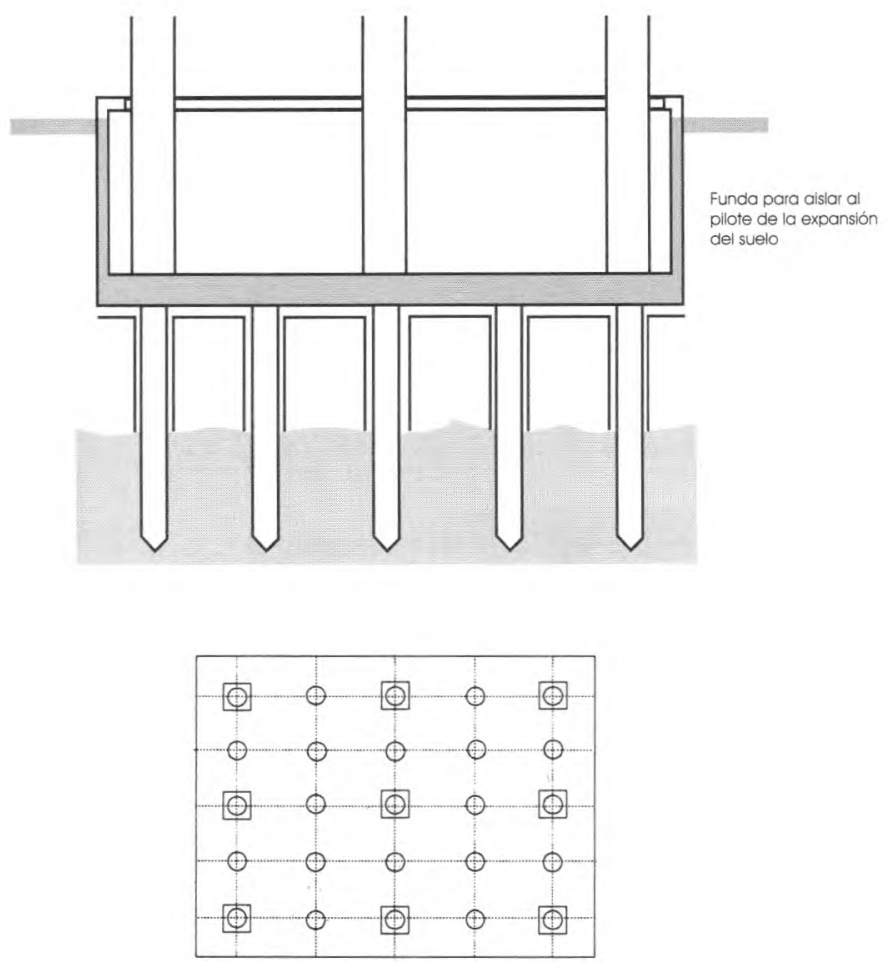

Figura 9. Cimentación con pilotes de fricción
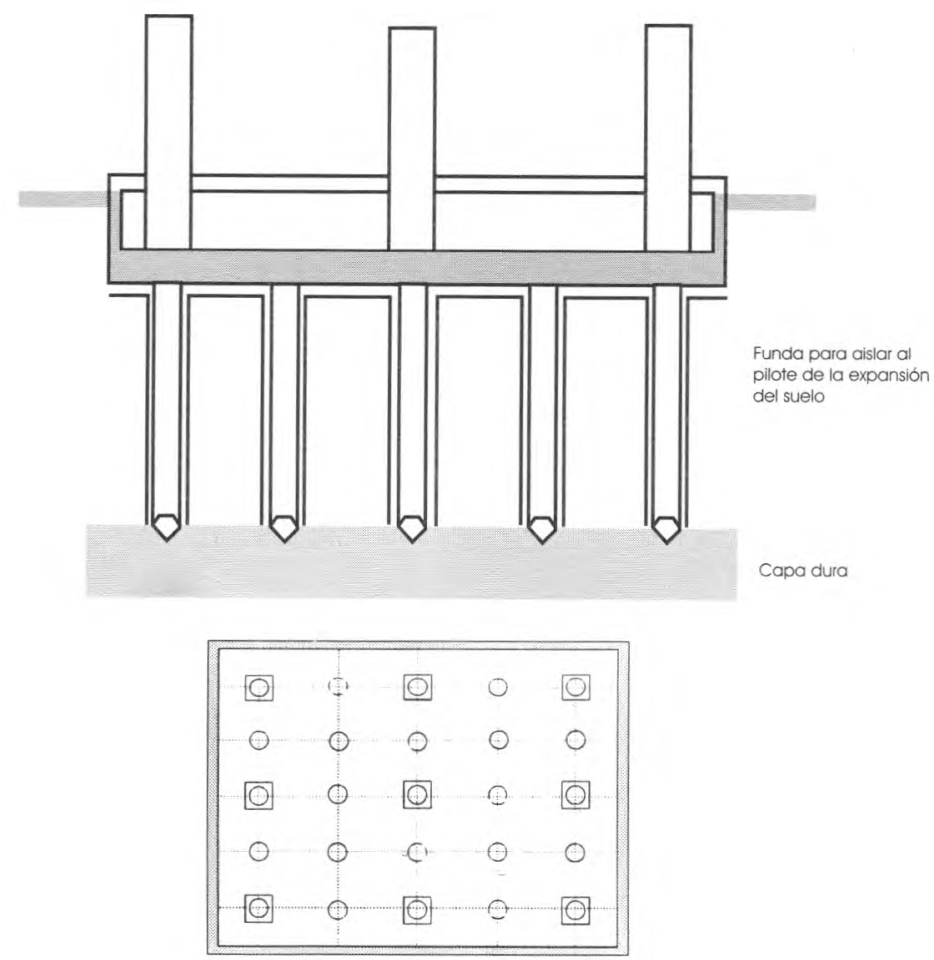

Figura 10. Cimentación con pilotes apoyados de punta 
En el caso de pilotes, se deberá colocar una funda para aislar al pilote de la zona de suelo expansivo.

El autor se verá recompensado si lo expuesto en este trabajo muestra utilidad en la práctica profesional y la motivación en los ingenieros estudiosos e investigadores en la disciplina racional de la mecánica de suelos para continuar la investigación que permita perfeccionar la tecnología de los suelos de características expansivas y así preveer o mitigar los daños que se ocasionan en las regiones donde la naturaleza ha formado este tipo de suelos.

\section{Referencias}

Grim R.E. (1953). Clay Mineralogy, cap.8. 161-162.

Grim R.E. (1948). Some Fundamental Factors Influencing the Properties of Soil Materials. Proc, Iconsomef. Vol. 3, p.8.
Rey A.E. (1982). Variación de los parámetros de resistencia con la humedad y el confinamiento del suelo. Tesis de maestria. DIMEI, Facultad de Ingeniería, UNAM.

Sánchez N.F. (1979). Estudio de un suelo expansivo de Coro, Venezuela. Tesis de maestria. DIMEI, Facultad de Ingeniería, UNAM.

Terzaghi K. (1941) Undistubed Clay Samples and Undistubed Clays. Journal of the Boston Society of Civil Engineers. No. 324.

Zeevaert-Wiechers L. (1982). Foundation Engineering for Difficult Subsoil Condittions. Van-Nostrand-Reinhold. Nueva York.

Zeevaert-Wiechers L. (1991). Endurecimiento de las arcillas. Simposio en la Sociedad Mexicana de Mecánica de Suelos, Agosto.

Zeevaert-Wiechers L. (1980). Interacción suelo-estructura de cimentaciones. Limusa. México.

\section{Semblanza del autor}

Leonardo Zeevaert-Wiechers. Obtuvo el título como ingeniero civil en 1939 en la Escuela Nacional de Ingenieros de la UNAM. Estudió el posgrado en el Instituto Tecnológico de Massachusetts donde recibió el grado de maestro en ingeniería en 1940. En 1943 inició una estrecha colaboración con el Dr. Karl Terzaghi en una investigación acerca de la estabilidad de las cortinas de corazón hidráulico construidas en México a principios de siglo XX, lo llevó en 1947 a trabajar en problemas especiales de mecánica de suelos en la Universidad de Illinois, donde obtuvo el grado de doctor (Ph. D) en 1949. Ha recibido numerosos reconocimientos, entre ellos: la medalla de oro profesional otorgada por el Instituto Americano de Arquitectos, diploma a la innovación tecnológica, la elección de profesor emérito y miembro de la Academia Nacional de Ingeniería de EUA, entre otros. El buen comportamiento de obras de ingeniería que ha diseñado, así como la cimentación y estructura de la Torre Latinoamericana, en donde introdujo el concepto de flexibilidad controlada en edificios altos, le ha valido para su reconocimiento a nivel internacional. Ha escrito 160 artículos, una gran cantidad de libros y ha presentado ponencias relacionadas con mecánica de suelos, ingeniería de cimentaciones y sísmica de cimentaciones. 


\section{Instructions for authors}

\section{Purpose and scope of the magazine}

The purpose of Ingenieria. Investigación y Tecnologia magazine is to spread original and unpublished information and knowledge related to all fields of engineering. It provides a forum to professors, researchers, practitioners and students from all over the world to submit for publication articles in either Spanish or English.

\section{The contributions will be classified in either one of the three following sections.}

Engineering in Mexico and around the world. Reviews and discussions related to the development, evolution and progress made in engineering issues of technical, scientific, epistemological and professional nature.

Recent studies and research. Papers written with a methodological, analytical, numerical and experimental approach as well as those characterized by technological innovation and development of practical application to education, professional activities and research work related to engineering.

Engineering and society. Analysis and discussion of social and professional issues related explicitly to engineering.

\section{Manuscripts}

1. The final original manuscripts properly written in Spanish or English should be submitted in a diskette with ASCII, Corel Ventura or Word format together with three hard legible copies with double-spaced printing and $3-\mathrm{cm}$ wide all-around margins. Illustrations should be submitted in ZIP encapsulated form indicating the format in which they were created.

2 . The heading of the article should include the following information: title of the paper; full name of author(s); present position; E-mail address, postal address, telephone and fax numbers of the author who will receive the correspondence; a list of keywords and a summary in both Spanish and English. The summary of no more than 200 words, should provide a concise, exact and unambiguous idea of the contents and scope of the article.

3. Sections and subsections of the article should be clearly differentiated but not numbered. Introduction: The subject, scope and purpose of the work should be described in lay language. Main body of the text: It should contain the plan of development of the subject matter in the paper, organized in a logic and simple manner; subdivisions of the text should include a heading without numbering. Conclusions: They should state concisely the contributions of the paper, as well as its scope, limitations and applicability. Acknowledgements: To be brief and concise. References and appendices.

4. The maximum length of the article should not exceed eight printed pages of text (equivalent to about 6500 words), or 10 pages including tables and figures.

5. Figures, photographs and tables should be provided as originals attached to the manuscript although not pasted into the text, but rather in separate folders and sheets and with dimensions compatible with the page size of the publication. The illustrations should be referred to as figures to be progressively numbered as mentioned in the text and identified as Fig. $1,2, \ldots$ or figure $1,2, \ldots$ Number tables in the same order in which they are mentioned in text and identify them as table $1,2, \ldots$

6. All mathematical expressions must be clearly typed, and care should be taken to differentiate the symbols unambiguously. It shall be compulsory to use the SI system of units.

7. For the references in the main body of the text give the last name(s) of the author(s) and the year of publication. For example: "Recently, Gracia-Sánchez and Estrada-Serrano (1996)..." or "In a recent paper (Gracia-Sánchez and Estrada-Serrano, 1996)..." All references appear together at the end of the paper listed alphabetically by last name of the first author according to the following general rules:

\section{Periodical publications}

Sotelo G. and Cafaggi A. (1996). Cálculo de la dimensión mínima de la sección de un canal o de los tirantes alternos, cuando se conocen el gasto y la energía específica. Ingeniería, Vol. LXVI, 25-46.

\section{Books}

Rosenblueth E. (1980). Sobre ciencia e ideología. Fundación Javier Barros Sierra, Mexico.

\section{Contributions to books}

Carrera-Bolaños J. (1994). Racionalidad, tecnología y desarrollo en: Los grandes problemas de la ciencia y la tecnología (Varela $\mathrm{R}$. and Mayer L. Ed.), pp.39-47. Universidad Autónoma Metropolitana, Mexico.

\section{Conference Reference}

Ayala G., Muñoz C. and Esteva L. (1984). Determination for Earthquake Design Parameters for Different Local and Topographic Conditions. $8^{\text {th }}$ World Conference on Earthquake Engineering, San Francisco California, USA, July.

\section{Unpublished reports/Thesis or dissertation}

Iturbe Argüelles M. del R. (1997). Transporte y dispersión de compuestos químicos orgánicos (hidrocarburos asfálticos halogenados) a través del flujo en medio poroso. PhD thesis in Engineering. Universidad Nacional Autónoma de México, Ciudad Universitaria, Mexico.

\section{Web sites}

Anderson S. (1997). Multimedia on the Internet: A course designed to study the tools of multimedia on the Word Wide Web. http://www.usu.edu/ sanderso/multinet/

8. A brief résumé of no more than 100 words should be included for each of the authors.

\section{Arbitration}

All articles submitted to the magazine should be reviewed by three arbitrators who will evaluate them and give an opinion about their publication. The Task Committee is integrated by top specialists with wide recognition in the different areas of expertise and trained to provide a reliable and expeditious evaluation of the quality and originality as well as of the value and interest of the paper contents.

\section{Proofreading of galleys}

Authors will receive the dummy of their articles for a final proofreading, together with a format of "assignment of copyright ownership and the right to reprint the articles".

\section{Reprints}

Ten copies of each article published will be sent on a complimentary basis to the senior author.

\section{Mailing of manuscripts}

The delivery of an article binds the author(s) to refrain for submitting it simultaneously for review at any other magazine or journal.

Manuscripts should be submitted for review by mailing them to the following address: Edificio " $A$ " de la Dirección, Facultad de Ingeniería, UNAM, Ciudad Universitaria, 04510 México, D.F., MEXICO, or to the following P.O. Box: Revista Ingeniería, Apartado Postal 70-402, Administración de Correos "Ciudad Universitaria", 04511 México, D.F., México. 